\title{
Fibrotic Scar After Spinal Cord Injury: Crosstalk With Other Cells, Cellular Origin, Function, and Mechanism
}

\author{
Ziyu $\mathrm{Li}^{\dagger}$, Shuisheng $\mathrm{Yu}^{\dagger}$, Xuyang Hu, Yiteng Li, Xingyu You, Dasheng Tian, Li Cheng*, \\ Meige Zheng* and Juehua Jing* \\ Department of Orthopaedics, The Second Hospital of Anhui Medical University, Hefei, China
}

\section{OPEN ACCESS}

Edited by:

Ya-Zhou Wang,

Fourth Military Medical

University, China

Reviewed by:

Byung Gon Kim

Ajou University, South Korea

Walace Gomes-Leal,

Federal University of Western

Pará, Brazil

*Correspondence:

Juehua Jing

jjhhu@sina.com

Meige Zheng

zhengmg113@126.com

Li Cheng

chengli7788@163.com

${ }^{\dagger}$ These authors have contributed equally to this work

Specialty section: This article was submitted to Non-Neuronal Cells,

a section of the journal

Frontiers in Cellular Neuroscience

Received: 05 June 2021

Accepted: 28 July 2021

Published: 26 August 2021

Citation:

Li Z, YU S, Hu X, Li Y, You X, Tian D, Cheng L, Zheng $M$ and Jing J (2021) Fibrotic Scar After Spinal Cord Injury: Crosstalk With Other Cells, Cellular Origin, Function, and Mechanism. Front. Cell. Neurosci. 15:720938. doi: 10.3389/fncel.2021.720938
The failure of axonal regeneration after spinal cord injury (SCI) results in permanent loss of sensorimotor function. The persistent presence of scar tissue, mainly fibrotic scar and astrocytic scar, is a critical cause of axonal regeneration failure and is widely accepted as a treatment target for SCl. Astrocytic scar has been widely investigated, while fibrotic scar has received less attention. Here, we review recent advances in fibrotic scar formation and its crosstalk with other main cellular components in the injured core after $\mathrm{SCl}$, as well as its cellular origin, function, and mechanism. This study is expected to provide an important basis and novel insights into fibrotic scar as a treatment target for SCl.

Keywords: spinal cord injury, fibrotic scar, crosstalk, cellular origin, treatment strategy

\section{INTRODUCTION}

Fibrosis, which is defined as fibroblasts excessively depositing extracellular matrix (ECM) mainly composed of collagen (COL), fibronectin (FN), and laminin (LN), is a common reaction to injury and inflammation in the central nervous system (CNS) and peripheral organs (Bataller and Brenner, 2005; Lee and Kalluri, 2010; Travers et al., 2016; Lederer and Martinez, 2018; Mack, 2018; Dorrier et al., 2021). In the early phase of tissue injury, a fibrotic scar provides the necessary support structure for the injured area and maintains tissue integrity. Due to the persistent presence of fibrotic scar, the normal tissue structure is disordered, and organ function is affected to varying degrees (Rockey et al., 2015; Pardali et al., 2017; Dorrier et al., 2021). Therefore, interventions targeting fibrotic scar formation are expected to be a potential targeted treatment.

Spinal cord injury (SCI) results in a series of intricate pathological changes (Sofroniew, 2018; Tran et al., 2018). Generally, direct physical trauma leads to apoptosis and necrosis of various cells, which, in turn, triggers inflammatory and immune responses. Finally, the persistence of scar tissue and inflammatory cells hinders axonal regeneration and impairs functional recovery (Hara et al., 2017; Dias et al., 2018; Kobayakawa et al., 2019). The scar tissue that forms after SCI mainly includes astrocytic scar, fibrotic scar, and microglial scar (Bellver-Landete et al., 2019). After SCI, activated astrocytes gradually aggregate, overlap, and surround the edge of the injured core, forming an astrocytic scar that contributes to limiting inflammation and promoting tissue retention, ultimately inhibiting axonal regeneration and resulting in permanent functional deficits (Wanner et al., 2013; Lang et al., 2015; Anderson et al., 2016; Hara et al., 2017; Tran et al., 2018). Contemporaneously, fibroblasts gradually proliferate, migrate, and corral a large number of macrophages in the injured core, forming a fibrotic scar adjacent to the medial side of the astrocytic scar (Göritz et al., 2011; Soderblom et al., 2013; Zhu et al., 2015a). The fibrotic scar is thought to maintain tissue integrity, limit inflammation, and inhibit axonal regeneration after SCI (Klapka et al., 2005; Dias et al., 2018; Wang et al., 2018a,b). The microglial scar located between the astrocytic scar and fibrotic scar is 
another glial scar component that was recently proposed and contributes to limiting inflammation, promoting wound healing, and enhancing functional recovery (Bellver-Landete et al., 2019). In the injured core after SCI, the area occupied by fibroblasts is larger than that occupied by the microglia, and the number of fibroblasts is approximately twice that of astrocytes (Göritz et al., 2011; Bellver-Landete et al., 2019). The ablation of astrocytic scar or microglial scar exerts adverse rather than beneficial effects on inflammation resolution and wound contraction, while moderately attenuating fibrotic scar may dramatically facilitate axonal regeneration and functional recovery after SCI (Herrmann et al., 2008; Renault-Mihara et al., 2017; Wang et al., 2018a,b; Bellver-Landete et al., 2019). However, most studies on scar tissue have focused on fibrotic scar in neurodegenerative disease or on astrocytic scar in SCI, and fibrotic scar after SCI has received little attention (Zhang et al., 2005; Kostyk et al., 2008; D’Ambrosi and Apolloni, 2020). Recently, Corey R. Fehlberg et al. and David Oliveira Dias et al. reviewed the formation, origin, and function of fibrotic scar in the CNS, providing new perspectives for the treatment of SCI (Dias and Göritz, 2018; Fehlberg and Lee, 2021). However, the spatiotemporal distribution of astrocytes, fibroblasts, macrophages, and microglia is closely related after SCI, suggesting a complex crosstalk between the main cellular components and fibrotic scar in the injured core, which has not been reviewed.

Therefore, the present review aims to summarize novel and different perspectives for an understanding of the formation of fibrotic scar and its crosstalk with other main cellular components, as well as its cellular origin, function, and mechanism. This study is expected to provide an important basis and novel insights into the fibrotic scar as a treatment target for SCI.

\section{FIBROTIC SCAR FORMATION AFTER SPINAL CORD INJURY}

After SCI, fibrotic scar is mainly composed of fibroblasts and excess ECM, mainly including COL, FN, and LN (Camand et al., 2004; Okada et al., 2007; Schiwy et al., 2009; Soderblom et al., 2013; Zhu et al., 2015a; Yokota et al., 2017). However, the formation and distribution characteristics of fibrotic scar vary slightly among species or SCI models.

Due to high clinical similarity and high reproducibility, the mouse spinal cord hemisection model and contusion model are commonly used as the disease models of SCI (Soderblom et al., 2013; Dias et al., 2018; Bellver-Landete et al., 2019; Kobayakawa et al., 2019). In the spinal cord hemisection injury model, fibroblasts begin to proliferate, migrate, and aggregate in the injured area at 3 days postinjury (dpi) (Göritz et al., 2011). The number of fibroblasts increases more than 25-fold at $9 \mathrm{dpi}$ and peaks at 14 dpi (Göritz et al., 2011). Meanwhile, fibroblasts aggregating in the injured area deposit a large amount of fibrous ECM and form a fibrotic scar adjacent to the medial side of the astrocytic scar, thus, filling the entire injured core, and the distributions of FN and LN overlap with fibroblasts (Göritz et al.,
2011; Soderblom et al., 2013; Dias et al., 2018). As the fibrotic scar contracts, the number of fibroblasts begins to decrease at 4 months and then is maintained for at least 7 months after SCI (Göritz et al., 2011). In the spinal cord contusion model, similar to the spinal cord hemisection model at $3 \mathrm{dpi}$, fibroblasts begin to proliferate, migrate, and aggregate in the injured area (Göritz et al., 2011; Soderblom et al., 2013). The cell density increases significantly at $5 \mathrm{dpi}$ and peaks at $7 \mathrm{dpi}$ (Soderblom et al., 2013). In addition, as fibroblasts proliferate and aggregate, FN secreted by fibroblasts initially exists in a soluble form and begins to assemble into ECM at 7 dpi (Zhu et al., 2015b). At $14 \mathrm{dpi}$, fibroblasts deposit a large amount of fibrous ECM, such as FN, which becomes much more organized into a fibrillar network, forming a dense fibrotic scar adjacent to the medial side of astrocytic scar (Soderblom et al., 2013; Zhu et al., 2015b). However, unlike the fibrotic scar, which fills the entire injured core and is mixed with macrophages after hemisection injury, the fibrotic scar that forms after contusion injury exhibits a dense and contiguous scar structure at the edge of the injured core filled with a large number of macrophages (Figure 1; Göritz et al., 2011; Soderblom et al., 2013). In addition, the distribution of FN overlaps with fibroblasts, while the distribution of LN is mainly located around the peripheral rim of the injured core (Göritz et al., 2011; Soderblom et al., 2013; Zhu et al., 2015a; Dias et al., 2018; Li et al., 2021). In both injury models, fibroblasts begin to proliferate and migrate at $3 \mathrm{dpi}$ and form mature and stable fibrotic scar structures at $14 \mathrm{dpi}$. Although differences in the spatial distribution of fibrotic scars in the two injury models have been identified, the similarity in the temporal distribution provides an important basis for subsequent research on fibrotic scar.

Consistent with the mouse spinal cord contusion model, the fibrotic scar also forms in a rat spinal cord contusion model, but the fibrotic scar distribution in the two species is slightly different (Ruschel et al., 2015; Zhu et al., 2015b). In contrast to mice, spinal cord contusion in rats leads to cavity formation in the injured core, which is considered to resemble the pathological changes observed in patients with SCI in the clinic (Metz et al., 2000; Norenberg et al., 2004; Buss et al., 2007). The existence of cavities after SCI in rats results in the fibrotic scars occupying a smaller area of the injured core than in mice, and the fibrotic scars are distributed along the edge of the cavity and partially overlap with astrocytic scars, indicating that astrocytes may be involved in the formation of fibrotic scars in rats and that differences may exist in the mechanism of scar formation between the two species (Zhu et al., 2015b). Considering the genetic homology with humans and the use of transgenic technology, a mouse model is still most commonly used to study SCI. However, advances in the treatment of SCI in mouse models should be further validated in rat models due to the cavity formation after SCI in rats, which is presumed to resemble patients with SCI in the clinic.

Overall, fibroblast proliferation and migration, and ECM deposition are important steps in fibrotic scar formation, and the temporal and spatial characteristics of fibrotic scar formation are of guiding significance for the formulation of subsequent research protocols. Studies aiming to further investigate the differences and commonalities of fibrotic scar among different 


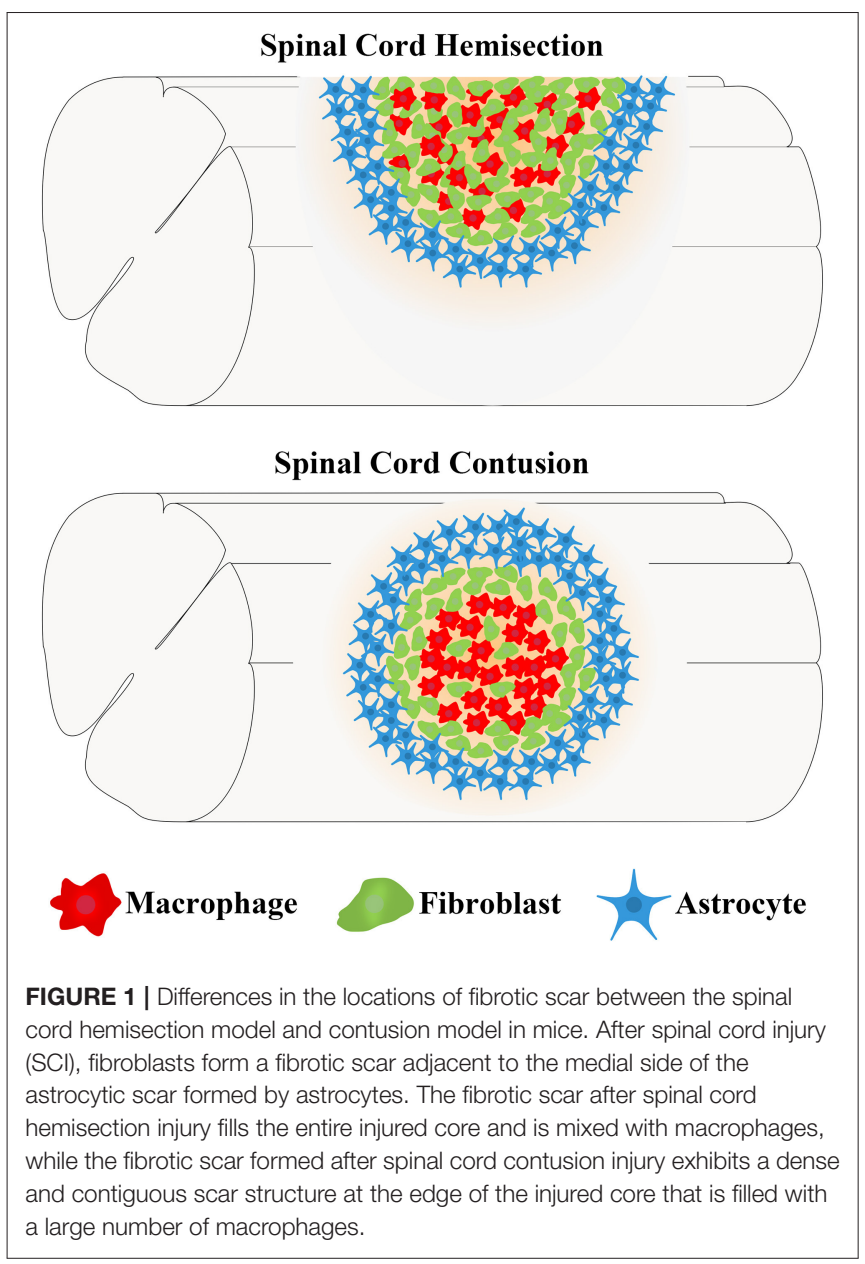

species and injury models after SCI are very important to further understand the cellular origin, function, and mechanism of fibrotic scar, providing important bases for their use as a treatment target for SCI.

\section{CROSSTALK BETWEEN THE FIBROTIC SCAR AND OTHER CELLS AFTER SPINAL CORD INJURY}

The main cells in the injured area, including astrocytes, microglia, fibroblasts, and macrophages, work together to form complex, mature, and stable structures that fill the entire injured core and are maintained in the chronic phase of SCI (Soderblom et al., 2013; Zhu et al., 2015a; Anderson et al., 2016; Bellver-Landete et al., 2019; Zhou et al., 2020). The fibrotic scar is adjacent to the medial side of the astrocytic scar and corrals the macrophages in the injured core, while microglia are located between the astrocytic scar and fibrotic scar (Figure 2; Bellver-Landete et al., 2019). Studies focused on the closely related spatiotemporal distribution indicate a functional crosstalk between the main cellular components in the injured core (Dias et al., 2018; BellverLandete et al., 2019; Kobayakawa et al., 2019).
A

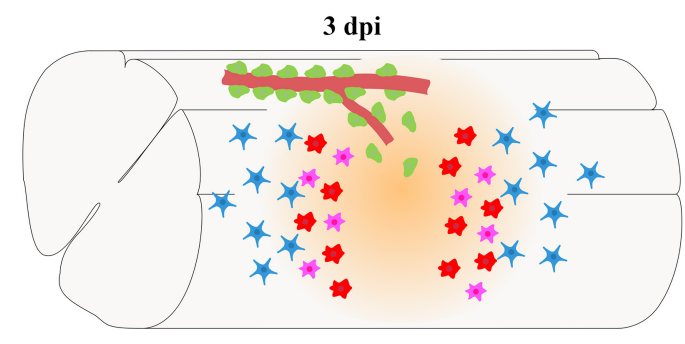

B

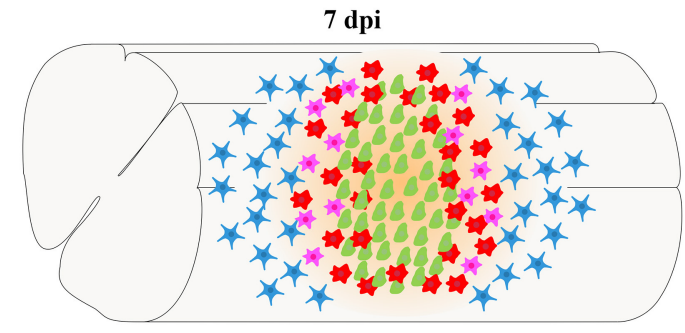

C

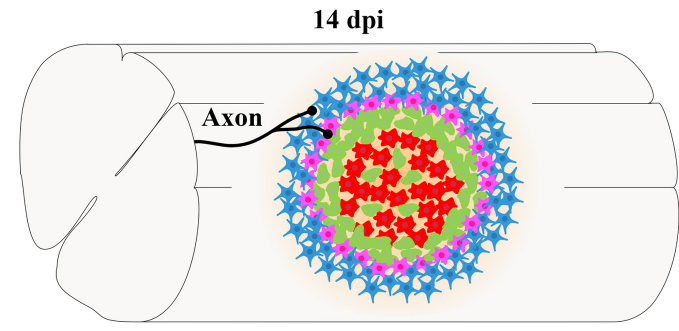

Blood Vessel $\longrightarrow$ Retraction Bulb

Astrocyte Microglia

Fibroblast

Macrophage

FIGURE 2 | The spatiotemporal distribution of scars after spinal cord injury. After SCl, astrocytes, microglia, fibroblasts, and macrophages contemporaneously proliferate, migrate, and aggregate at the injured core. Finally, the cells work together to form complex, mature, and stable structures that fill the entire injured core, which is maintained in the chronic phase of $\mathrm{SCl}$. (A) At 3 dpi, fibroblasts leave the blood vessels and are dispersed in the injured area together with astrocytes, microglia, and macrophages. (B) At 7 $\mathrm{dpi}$, the numbers of fibroblasts, astrocytes, microglia, and macrophages are significantly increased, and these cells aggregate at the injured core. (C) At 14 dpi, astrocytes form the astrocytic scar that are distributed in the outer layer and surrounding the entire injured core. Fibroblasts form a fibrotic scar adjacent to the medial side of the astrocytic scar and corral the macrophages in the injured core. Microglia are located between the astrocytic scar and fibrotic scar, while macrophages are located at the most central site of the injured core. The axon tips form retraction bulbs after contacting fibroblasts and fail to penetrate the dense barrier formed by the fibrotic scar at the edge of the injured core, resulting in the failure of axonal regeneration.

\section{Crosstalk Between the Fibrotic Scar and Astrocytes}

Activated astrocytes deposit the ECM component chondroitin sulfate proteoglycans (CSPGs), forming an astrocytic scar that surrounds and limits the fibrotic scar and macrophages in the injured core after SCI (Wanner et al., 2013; Anderson et al., 2016). In particular, fibroblasts and astrocytes are adjacent to each other, suggesting that astrocytes may play an important 
role in the formation and function of a fibrotic scar. Signal transducer and activator of transcription 3 (STAT3) is a critical regulator of astrocytes, the formation of astrocytic scar, and corralling fibroblasts and macrophages after SCI (Herrmann et al., 2008; Wanner et al., 2013). GFAP-STAT3-CKO mice in which STAT3 is conditionally knocked out in astrocytes exhibit a loss of astrocyte hypertrophy and astrocytic scar disruption after SCI. Astrocytes at the edge of the injured core are dispersed and no longer overlap, resulting in the loss of the contiguous boundary of the fibrotic scar and the diffusion of macrophages (Herrmann et al., 2008; Wanner et al., 2013; Renault-Mihara et al., 2017). After the disruption of the astrocytic scar, the fibrotic scar alone does not completely limit the macrophages in the injured core after SCI, suggesting that studies based on limiting inflammation after SCI should consider the combined functions of the astrocytic scar and fibrotic scar. Moreover, GlastRasless transgenic mice in which the proliferation of fibroblasts is inhibited to attenuate fibrotic scarring after SCI exhibit a reduction in astrogliosis and disruption of the contiguous boundary of astrocytic scar (Dias et al., 2018). These results suggest a crosstalk between the astrocytic scar and fibrotic scar to maintain the structural stability of each scar type after SCI.

The ligand Ephrin-B2 and its cell membrane receptor EphB2 promote cell-cell contact and are bidirectionally activated via phosphorylation (Bundesen et al., 2003). Liza Q. Bundesen et al. reported that Ephrin-B2 expressed on astrocytes binds to EphB2 expressed on fibroblasts, triggering the formation of the boundary between astrocytic scar and fibrotic scar after SCI (Bundesen et al., 2003). Ephrin-B2-/- mice in which the Ephrin B2 gene was deleted under the GFAP promoter exhibit a reduction in astrogliosis, facilitation of axonal regeneration, and improvement in motor function (Ren et al., 2013). Furthermore, after using RNAi to inhibit the expression of EphB2, the formation of astrocytic scar and fibrotic scar is inhibited, promoting axonal regeneration and myelination (Wu et al., 2021). These results indicate that the crosstalk between fibroblasts and astrocytes mediated by Ephrin-B2 and EphB2 is a therapeutic target after SCI, but specific intracellular signaling pathways require further exploration. In addition, Yona Goldshmit et al. reported that EphA4-deficient mice exhibit reduced astrocytic scarring, improved axonal regeneration, and better motor function after SCI (Goldshmit et al., 2004), in contrast to the report that mice lacking EphA4 do not exhibit a reduction in astrocytic scar or disruption of the boundary between astrocytic scar and fibrotic scar after SCI (Herrmann et al., 2010). The role of EphA4 in the crosstalk between astrocytes and fibroblasts requires further investigation.

\section{Crosstalk Between the Fibrotic Scar and Macrophages}

Macrophages and fibroblasts are widely distributed in peripheral organs and the CNS, and crosstalk between macrophages and fibroblasts is involved in the maintenance of homeostasis under healthy conditions and in disease progression, especially the process of fibrosis (Cai et al., 2020; Buechler et al., 2021). However, the role and mechanism of macrophages in regulating fibrotic scar formation after SCI still remain to be explored. After SCI, blood-derived macrophages infiltrate the injured area and share many markers and behaviors with microglia, increasing the difficulty of distinguishing between macrophages and microglia (Wang et al., 2015). Recently, genetic fate mapping and conditional gene targeting technologies have allowed researchers to conclude that macrophages infiltrating from the circulatory system after SCI are mainly distributed in the injured core and limited by the fibrotic scar, while resident microglia are mainly localized at the lesion border and distributed between the astrocytic scar and fibrotic scar (Wang et al., 2015; Zhu et al., 2015a).

Macrophages and fibroblasts simultaneously proliferate, migrate, and aggregate at the injured core after SCI, and finally, a large number of macrophages fill in and are limited inside the mature fibrotic scar at 14 dpi (Zhu et al., 2015a,b). After the elimination of blood-derived macrophages in the injured core, the density of fibrotic scar is significantly reduced, and the contiguous boundary formed by the fibrotic scar is disrupted, suggesting that macrophages are involved in fibrotic scar formation by regulating fibroblast migration (Zhu et al., 2015a; Zhou et al., 2020). Further investigations of the cytokine expression profile after the elimination of blood-derived macrophages showed that the expression of a profibrotic molecule, a proliferation-inducing ligand (APRIL), was significantly decreased, while the expression of antifibrotic molecules and bone morphogenetic proteins (BMPs) was significantly increased (Zhu et al., 2015a). APRIL $\mathrm{KO}$ mice exhibit a reduction in the fibrotic scar area and an improvement in axonal regeneration after SCI, which may result from the reduced infiltration of $\mathrm{B}$ cells and macrophages (Funk et al., 2016). Therefore, the direct role of APRIL in the crosstalk between macrophages and fibroblasts requires further investigation, and BMPs and other potential molecular mechanisms should also be explored. In addition, integrin $\alpha 5 \beta 1$, which is mainly expressed on fibroblasts and macrophages, may be involved in the assembly of FN into the fibrotic scar by functioning as a receptor of FN after SCI, and the specific role of $\alpha 5 \beta 1$ requires further study to provide evidence for the crosstalk between macrophages and fibroblasts (Zhu et al., 2015b).

Similar to macrophages, neutrophils and $\mathrm{T}$ cells, which are inflammatory cells, are also involved in the inflammatory response after SCI, and their role in fibrotic scar formation should be considered in future studies (Li et al., 2018; Liu et al., 2019; Zivkovic et al., 2021). In a mouse multiple sclerosis (MS) model, with the infiltration of $\mathrm{T}$ cells, fibroblasts proliferate, aggregate, and deposit COL1 to form a fibrotic scar (Dorrier et al., 2021). The use of FTY720, which inhibits the exit of immune cells from lymph nodes and has been used to treat MS in the clinic, to inhibit the infiltration of immune cells, significantly results in the inhibition of fibroblast proliferation and COL1 deposition after MS (Dorrier et al., 2021). Therefore, the role of inflammatory cells other than macrophages in SCI, especially in fibrotic scarring, requires further attention. The effects of treatment strategies designed to suppress inflammation after SCI on the fibrotic scar should be considered, and further studies are needed to determine whether FTY720 and other CNS anti-inflammatory 
drugs that have been used in the clinic exert a therapeutic effect on SCI.

\section{Crosstalk Between the Fibrotic Scar and Microglia}

During the period of fibrotic scar formation after SCI, microglia gradually distributed between the astrocytic scar and fibrotic scar, forming a microglial scar (Wang et al., 2015; BellverLandete et al., 2019). The special spatial distribution indicates that microglia may play a critical role in the formation of astrocytic scar and fibrotic scar. The use of PLX5622, a highly selective inhibitor of the colony-stimulating factor 1 receptor, to deplete the microglia disrupts the density and contiguous boundary of the astrocytic scar and fibrotic scar, resulting in the spread of macrophages in the injured core and the impairment of locomotor recovery after SCI (Bellver-Landete et al., 2019). Based on these results, the microglia are essential for maintaining the stability of scars. The migration of the microglia to the edge of the injured core to form scars is mediated by IGF1, a microglia-derived factor, and the effect of IGF-dependent microglial migration on fibrotic scar formation after SCI and its mechanism require further exploration (Bellver-Landete et al., 2019).

Recently, Yi Li et al. reported that the microglia organize scarfree repair after SCI in neonatal mice, in significant contrast to persistent scars and failure of axonal regeneration in adult mice (Li et al., 2020). After SCI in neonatal mice, the microglia are the MG3 type, referred to as repair-promoting microglia that expressed proteinase inhibitors at high levels and transiently secreted FN to form effective bridges that connected the injured ends of the spinal cord. Finally, the nearly complete recovery of neonatal mice after SCI was observed, while macrophages, fibrotic scar, and astrocytic scar were absent (Li et al., 2020). After treatment with the chemical proteinase inhibitors E64 and serpin $\mathrm{A} 3 \mathrm{~N}$ and transplantation into the injured core of adult mice, adult mouse-isolated microglia exhibited a similar function to repair-promoting microglia, including less infiltration of macrophages, less deposition of COL, and more regenerated axons after SCI (Li et al., 2020). Therefore, repair-promoting microglia expressed higher levels of proteinase inhibitors, which have a critical role in resolving inflammation and inhibiting scar formation, thereby facilitating axonal regeneration. The role and mechanism of proteinase inhibitors in scar-free repair organized by microglia remain to be explored in both neonatal and adult mice, which may provide novel treatment strategies for SCI.

\section{CELLULAR ORIGIN OF THE FIBROTIC SCAR AFTER SPINAL CORD INJURY}

Previous electron microscopy studies have identified the presence of fibroblast-like cells in the contused spinal cord, and fibrotic scars have consistently been shown to form after SCI (Zhang et al., 2005; Kostyk et al., 2008). In the past, due to the limitation of a lack of specific markers for fibroblasts, the spatiotemporal distribution and origin of fibrotic scar-forming fibroblasts were unclear after SCI (Rudge and Silver, 1990; Shearer and Fawcett,
2001; Zeisberg and Kalluri, 2004; Darby and Hewitson, 2007; Krenning et al., 2010; Soderblom et al., 2013). Recently, using transgenic mice, Jonas Frisén et al. and Jae K. Lee et al. revealed the origin, phenotype, and distribution of fibrotic scar-forming fibroblasts after SCI (Göritz et al., 2011; Soderblom et al., 2013; Dias et al., 2018).

Pericytes, the perivascular cells associated with the microvascular system and wrapped around vascular endothelial cells may differentiate into fibroblasts and form a fibrotic scar in dermal scar and in kidney fibrosis (Sundberg et al., 1996; Lin et al., 2008; Humphreys et al., 2010). Jonas Frisén et al. used GLAST-CreER $^{\mathrm{T} 2}$ transgenic mice to identify GLAST ${ }^{+}$pericytes as type A pericytes and investigate their role in fibrotic scar formation after SCI (Srinivas et al., 2001; Slezak et al., 2007; Göritz et al., 2011). Blocking the proliferation of type A pericytes results in a significant reduction in, or even the disappearance of, fibrotic scar after SCI, suggesting that fibrotic scar-forming fibroblasts are derived from type A pericytes (Göritz et al., 2011). In the CNS, most or all pericytes are labeled with CD13, plateletderived growth factor receptor (PDGFR) $\alpha$ and PDGFR $\beta$, while some pericytes express desmin and $\alpha$-SMA (Bondjers et al., 2006; Armulik et al., 2010; Daneman et al., 2010; Göritz et al., 2011). In the uninjured spinal cord, type A pericytes, which are enfolded by the plasma membrane of astrocytes and basal lamina, are distributed away from the blood vessel wall compared with type B pericytes and express CD13, PDGFR $\alpha$, and PDGFR $\beta$ (Göritz et al., 2011). Type B pericytes express $\alpha$-SMA or desmin (Göritz et al., 2011). After SCI, type A pericytes leave blood vessels, proliferate, and migrate to the injured area, depositing ECM to form a fibrotic scar. At this time, these cells no longer express CD13 and PDGFR $\alpha$ but express PDGFR $\beta$ and the fibroblast marker FN and transiently express $\alpha$-SMA (Göritz et al., 2011). These results indicate that type A pericytes are the main source of fibrotic scar-forming fibroblasts that are specifically labeled with PDGFR $\beta$ after SCI.

Commonly used markers of fibroblasts, such as FN and COL, are components of the ECM and do not specifically label the cell body (Kalluri and Zeisberg, 2006). Jae K. Lee et al. used Col1 $\alpha 1$-GFP transgenic mice to specifically label fibroblasts in the spinal cord and revealed that PDGFR $\beta^{+}$perivascular fibroblasts are the main fibrotic scar-forming fibroblasts after SCI (Yata et al., 2003; Soderblom et al., 2013). In the uninjured spinal cord, Coll $\mathrm{\alpha l}^{+}$cells are mainly located around large-diameter blood vessels, and most of them surround smooth muscle cells in the arterioles, while $78.7 \%$ of Coll $\alpha 1^{+}$cells express CD13 and $95.7 \%$ of Col1 $\alpha 1^{+}$cells express PDGFR $\beta$ but not desmin and $\alpha$-SMA (Soderblom et al., 2013). After SCI, Coll $\alpha 1^{+}$cells leaving blood vessels proliferate, migrate, and aggregate at the edge of the injured core, depositing ECM to form a fibrotic scar around the injured core, where $26.9 \%$ of Col $\alpha 1^{+}$cells express $\alpha$-SMA, $26.9 \%$ of Col $\alpha 1^{+}$cells express CD13, and $100 \%$ of Coll $\alpha 1^{+}$cells express PDGFR $\beta$ (Soderblom et al., 2013). As before injury, Coll $\alpha 1^{+}$cells still do not express desmin, $\alpha$-SMA, or other markers of perivascular cells, including NG2, Olig2, and GFAP (Soderblom et al., 2013). Thus, Coll $\alpha 1^{+}$cells are perivascular fibroblasts forming a fibrotic scar that specifically express PDGFR $\beta$ after SCI (Zhu et al., 2015b; Funk et al., 2016). 
Michael Vanlandewijck et al. performed a single-cell sequencing study that provided molecular definitions of blood vessels and blood vessel-related cells in the CNS of mice and revealed a population of PDGFR $\alpha^{+}$fibroblast-like cells; many of the fibroblast-specific transcripts encode collagens, collagenmodifying enzymes, and proteins involved in collagen fibril assembly, indicating the fibrosis-promoting characteristic of fibroblasts (Vanlandewijck et al., 2018). PDGFR $\alpha^{+}$fibroblasts are distributed around the large-diameter blood vessels in the meninges, parenchyma, and choroidal plexus (Vanlandewijck et al., 2018), consistent with the distribution of Glast ${ }^{+}$or Col $1 \alpha 1^{+}$fibroblasts reported by Jonas Frisén et al. and Jae K. Lee et al. (Göritz et al., 2011; Soderblom et al., 2013). However, PDGFR $\alpha^{+}$fibroblasts are located between the vascular wall and astrocyte end-feet (Vanlandewijck et al., 2018), which is different from the study by Jonas Frisén et al., who reported that Glast ${ }^{+}$ fibroblasts are enfolded by the plasma membrane of astrocytes and the basal lamina (Göritz et al., 2011). Indeed, both Glast and PDGFR $\beta$ are expressed at high levels in fibroblasts and pericytes, suggesting that Glast ${ }^{+}$fibroblasts and $\operatorname{Col} 1 \alpha 1^{+}$fibroblasts are essentially perivascular fibroblasts, as reported by Vanlandewijck et al. (2018) and Dorrier et al. (2021). Recently, Cayce E. Dorrier et al. examined an MS model and reported that fibrotic scar is formed by Coll $\alpha 1^{+}$fibroblasts, which are initially distributed around large-diameter blood vessels and express PDGFR $\alpha$ and PDGFR $\beta$ (Dorrier et al., 2021). Although the localization of fibroblasts remains controversial, the fibrotic scar is formed from large-diameter perivascular fibroblasts that are labeled with CD13, PDGFR $\beta$, and PDGFR $\alpha$ before injury and specifically labeled with PDGFR $\beta$ after SCI. Further study is needed to confirm this hypothesis.

\section{FUNCTION OF THE FIBROTIC SCAR AFTER SPINAL CORD INJURY}

Scars, mainly astrocytic scar, fibrotic scar, and microglial scar, are important pathological changes in the chronic stage after SCI (Soderblom et al., 2013; Anderson et al., 2016; Bellver-Landete et al., 2019). The astrocytic scar has been widely studied and is considered to inhibit inflammation, maintain tissue integrity, and inhibit axonal regeneration (Anderson et al., 2016; Sofroniew, 2018; Xie et al., 2020). Indeed, the fibrotic scar also plays a dual function after SCI and should receive more attention (Hermanns et al., 2001; Göritz et al., 2011; Dias et al., 2018).

In the chronic phase of SCI, axon tips form retraction bulbs after contacting fibroblasts and fail to penetrate the dense barrier formed by the fibrotic scar at the edge of the injured core, resulting in the failure of axonal regeneration (Figure 2C; Dias et al., 2018). Nicole Klapka et al. reported that inhibiting TGF $\beta 1$ leads to delayed fibrotic scar formation after SCI, and regenerated CST axons pass through the damaged core, resulting in improved axonal regeneration and motor function (Klapka et al., 2005). Subsequent studies further confirmed that the reduction or disruption of fibrotic scar after SCI increases the density of regenerated axons in the damaged core, promoting the recovery of motor function (Hellal et al., 2011; Ruschel et al., 2015; Yokota et al., 2017; Cooper et al., 2018). Recently, Jonas Frisén et al. used Glast-Rasless transgenic mice to inhibit the proliferation of type A pericytes and specifically block pericyte-derived fibrotic scar after SCI; the results showed that attenuating fibrotic scar formation led to a reduction in astrogliosis, diminished astrocyte reactivity, disruption of the contiguous boundary of astrocytic scar, regeneration of 5-HT, and CST axons were enhanced, and the recovery of sensorimotor function was improved in the chronic phase of SCI (Dias et al., 2018). Therefore, the fibrotic scar significantly inhibits axonal regeneration in the chronic phase of SCI, and preventing fibrotic scar formation is an important direction for the treatment of SCI.

However, the inhibition of fibrotic scar formation did not significantly improve motor function despite the promotion of axonal regeneration in other studies (Zhu et al., 2015a). Jonas Frisén et al. showed that completely blocking the fibrotic scar results in the failure of wound closure and the spread of macrophages in the injured core at 14 dpi (Göritz et al., 2011; Dias et al., 2018). Therefore, in addition to inhibiting axonal regeneration in the chronic phase of SCI, fibrotic scarring also exerts beneficial effects on maintaining tissue integrity and limiting inflammation in the early phase of SCI. Treatment targeting the fibrotic scar should be based on the dual function of fibrotic scar in different phases of SCI, and interventions promoting the formation of a fibrotic scar in the early phase while inhibiting the formation of the fibrotic scar in the chronic phase may exert a better therapeutic effect.

\section{MECHANISM OF FIBROTIC SCAR FORMATION AFTER SPINAL CORD INJURY AND TREATMENT STRATEGIES}

The fibrotic scar is recognized as a treatment target in many diseases, including SCI (Pardali et al., 2017; Dias et al., 2018; Dorrier et al., 2021). Therefore, a review of the mechanisms of the fibrotic scar formation may provide novel insights into the role of the fibrotic scar as a treatment target after SCI. Migration, proliferation, and ECM deposition are the key processes fibroblasts use to form fibrotic scar (Göritz et al., 2011; Soderblom et al., 2013; Dias et al., 2018), of which the mechanisms should receive more attention and be well-reviewed.

\section{Transforming Growth Factor Beta 1}

Transforming growth factor beta 1 (TGF- $\beta 1$ ) is a profibrotic factor that regulates fibroblast proliferation and ECM deposition in the CNS (Kawano et al., 2012; Wang et al., 2018a). The expression levels of microRNA-21-5p and TGF- $\beta 1$ are significantly increased after SCI (Wang et al., 2018a). TGF$\beta 1$ promotes the expression of microRNA-21-5p and fibrosisassociated genes such as FN and COL in fibroblasts, while the upregulation of microRNA-21-5p enhances the profibrotic activity of TGF- $\beta 1$ toward fibroblasts in vitro (Wang et al., $2018 \mathrm{a}, \mathrm{b})$. Based on these results, microRNA-21-5p enhances TGF- $\beta 1$ activity in an amplifying circuit, promoting fibroblastmediated formation of fibrotic scar after SCI. In addition, intrathecal injection of antagomir-21 was used to establish 
a microRNA-21-5p knockdown mouse model, resulting in improved functional recovery after SCI (Wang et al., 2018a,b). However, animal studies are needed to further confirm that microRNA-21-5p targets TGF- $\beta 1$ to regulate the formation of a fibrotic scar after SCI.

Bradke et al. reported that SCI mice treated with the microtubule-stabilizing agents Taxol and epothilone $\mathrm{B}$ (epoB) exhibit enhanced axonal regeneration and the inhibition of fibrotic scar formation partially through dampening of the TGF $\beta 1 /$ Smad2 pathway, which is conducive to functional recovery after SCI (Hellal et al., 2011; Ruschel et al., 2015). Nicole Klapka et al. treated SCI rats with 8-Br-cAMP to inhibit the fibrotic effect of TGF- $\beta 1$ and the iron chelator BPY-DCA to inhibit prolyl 4-hydroxylase, resulting in delayed COL IV expression and fibrotic scar formation (Hermanns et al., 2001; Klapka et al., 2005). The treatment improved axonal regeneration and motor function recovery (Hermanns et al., 2001; Klapka et al., 2005). In summary, TGF- $\beta 1$ plays critical roles in fibrotic scar formation after SCI and can be used as a treatment target. Further studies examining the source of TGF- $\beta 1$ and the molecular mechanism of the TGF- $\beta 1$ pathway may be very important to reveal novel treatment targets for SCI.

\section{Periostin}

Periostin (POSTN), an ECM protein, is a member of the fasciclin family that plays critical roles in the development of the heart, tooth, and bone tissue (Hakuno et al., 2010). POSTN also regulates fibrosis in many organs, such as the lungs, liver, and

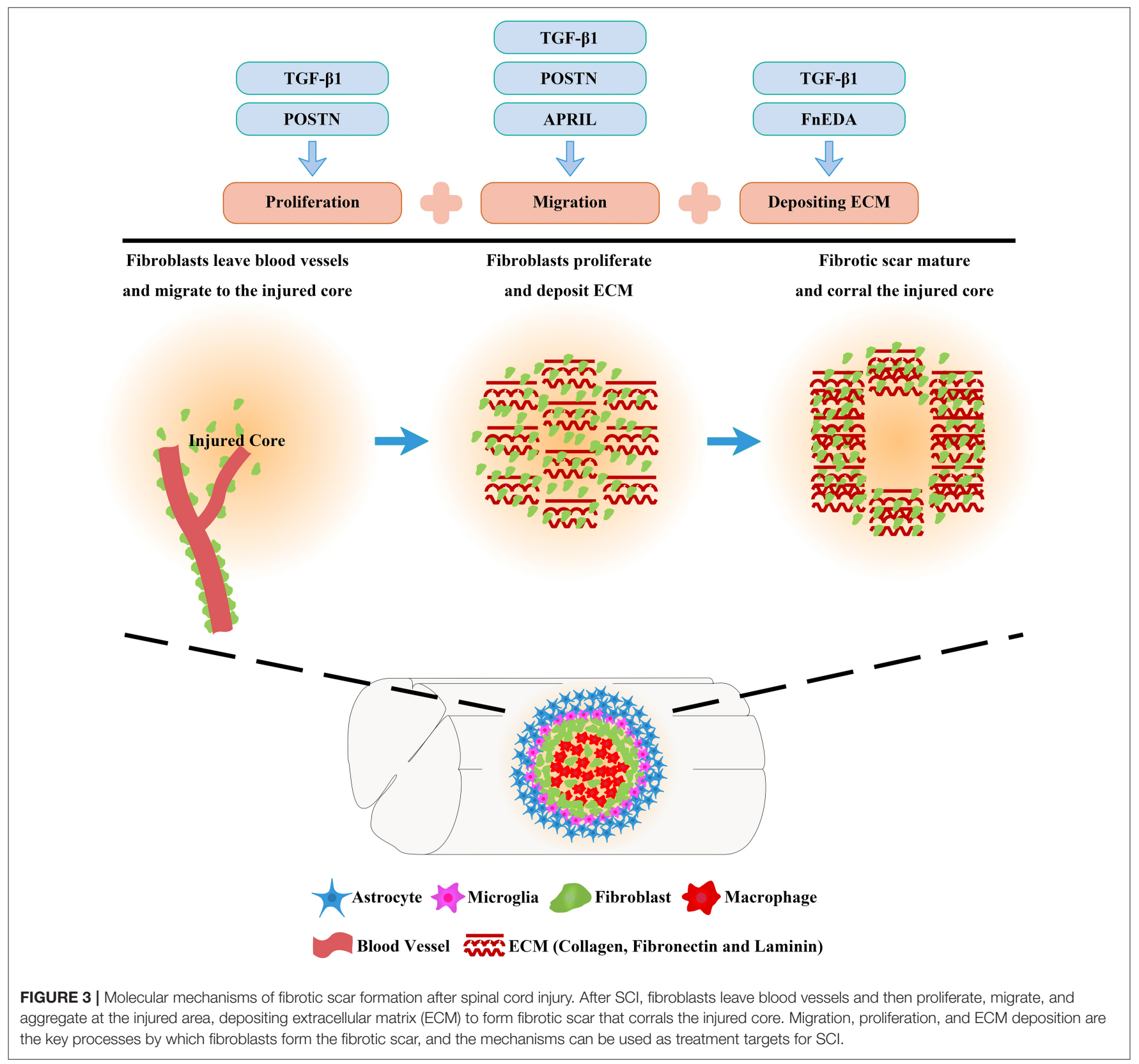


skin (Yamaguchi, 2014; Izuhara et al., 2016; Sugiyama et al., 2016). In particular, POSTN is specifically overexpressed in PDGFR $\beta^{+}$cells referred to as fibroblasts after SCI, indicating that POSTN may regulate fibrotic scar formation after SCI (Yokota et al., 2017). In POSTN KO mice, the proliferation of fibroblasts is significantly inhibited after SCI, resulting in reduced fibrotic scarring, increased axonal regeneration, and improved functional recovery (Yokota et al., 2017). Further studies revealed that POSTN promotes macrophage infiltration to recruit fibroblasts to the injured area after SCI and increases the secretion of $\mathrm{TNF} \alpha$ by macrophages to promote fibroblast proliferation (Yokota et al., 2017). Strikingly, mice receiving an injection of a POSTN-neutralizing antibody after SCI shows reduced fibrotic scarring and better functional recovery (Yokota et al., 2017). Thus, POSTN, specifically expressed in fibroblasts, is a potential profibrotic molecule that may promote fibroblast proliferation and migration, leading to fibrotic scar formation. Further studies investigating the role and molecular mechanism of POSTN in SCI may provide more evidence for targeted therapy for SCI.

\section{A Proliferation-Inducing Ligand}

APRIL is well-known as a regulator of B-cell maturation and survival that is expressed in macrophages, $\mathrm{B}$ cells, and activated $\mathrm{T}$ cells (Chan et al., 2021). APRIL plays important roles in various diseases of peripheral organs and the CNS, such as rheumatoid arthritis and multiple sclerosis (Wang et al., 2012; Weldon et al., 2015; Funk et al., 2016). The depletion of macrophages in the injured core after SCI results in a reduction in the fibrotic scar density and the disruption of the contiguous boundary of fibrotic scar, promoting axonal regeneration (Zhu et al., 2015a). Therefore, treatment strategies that suppress inflammation may play additional roles in SCI by inhibiting fibrotic scar formation. In addition, after the depletion of macrophages, the reduction in fibrotic scarring is associated with the downregulation of APRIL, and the expression of APRIL and its receptor, B-cell maturation antigen (BCMA), is dramatically upregulated after SCI, suggesting that APRIL may be involved in fibrotic scar formation (Zhu et al., 2015a; Funk et al., 2016). In APRIL KO mice, the infiltration of $B$ cells and macrophages is substantially reduced after SCI, resulting in a reduction in fibrotic scar area but no effect on fibroblast proliferation, and the effect of APRIL on macrophage and B-cell infiltration may be mediated by the upregulation of the expression of TNF- $\alpha$ and CCL2 (Funk et al., 2016). These results suggest that APRIL is a profibrotic molecule that is involved in the recruitment of fibroblasts by regulating the infiltration of macrophages and B cells after SCI.

\section{Fibronectin Including the Extra Domain A}

The assembly of the FN matrix, a component of fibrotic scar, is an important step in the formation of a fibrotic scar after SCI, while the isoform of $\mathrm{FN}$ including the extra domain A domain (FnEDA) is involved in the pathogenesis of fibrotic scar in various diseases (Bhattacharyya et al., 2013; Zhu et al., 2015b; Cooper et al., 2018). The expression of FnEDA is significantly increased and that protein is chronically deposited in the injured core after SCI (Cooper et al., 2018). In FnEDA-null mice, the expression level of insoluble FN and the area of fibrotic scar are decreased significantly at 90 days after SCI, leading to improved axonal regeneration and functional recovery (Cooper et al., 2018). These results suggested a critical role for FnEDA in the assembly of fibrotic scar in the chronic phase after SCI and its potential use as a treatment target for SCI.

\section{Wnt}

The Wnt pathway plays an indispensable role in disease processes in the CNS (Lie et al., 2005; Wang et al., 2011). The activity of Wnt signaling after SCI can be detected by performing Xgal staining in Wnt signaling reporter TOPgal transgenic mice (DasGupta and Fuchs, 1999; Yamagami et al., 2018). The Wnt/ $\beta$ catenin pathway is transiently activated in $\mathrm{FN}^{+}$fibroblasts in the injured core after SCI (Yamagami et al., 2018). Thus, the Wnt/ $\beta$ catenin pathway may be involved in the formation of a fibrotic scar after SCI, which should be further investigated to provide a novel treatment target for SCI.

In addition, strategies such as neural stem cell (NSC) transplantation for SCI significantly promote axonal regeneration and functional recovery, suggesting that NSCs may be able to overcome the effects of adverse factors, including the fibrotic scar. The interaction between the two is worth further

TABLE 1 | Treatments targeting the formation of fibrotic scar after spinal cord injury (SCI).

\begin{tabular}{llll}
\hline Treatment strategy & Target & Effect on fibroblasts & References \\
\hline Antagomir-21 & TGF- $\beta 1$ & Depositing ECM $\downarrow$ & Wang et al., 2018a,b \\
EpoB & TGF- $\beta 1 /$ Smad2 & Migration and depositing ECM $\downarrow$ & Ruschel et al., 2015 \\
Taxol & TGF- $\beta 1 /$ Smad2 & Migration and depositing ECM $\downarrow$ & Hellal et al., 2011 \\
8-Br-CAMP & TGF- $\beta 1$ & Depositing ECM $\downarrow$ & Klapka et al., 2005 \\
POSTN-neutralizing antibody & POSTN & Proliferation and migration $\downarrow$ & Yokota et al., 2017 \\
APRIL KO mice & APRIL/BCMA & Migration $\downarrow$ & Funk et al., 2016 \\
FnEDA-null mice & FnEDA & Depositing ECM $\downarrow$ & Cooper et al., 2018 \\
Clodronate liposomes & Macrophage & Migration $\downarrow$ & Zhu et al., 2015a \\
Glast-Rasless transgenic mice & Ras & Proliferation $\downarrow$ & Dias et al., 2018
\end{tabular}

EpoB, epothilone B; TGF-B1, transforming growth factor beta 1; ECM, extracellular matrix; POSTN, periostin; APRIL, a proliferation inducing ligand; BCMA, B cell maturation antigen; FnEDA, fibronectin including the extra domain $A$. 
study. Mark H. Tuszynski et al. transplanted NSCs into rats or a primate model of SCI and showed that NSC-differentiated neurons extended axons through the injured core and filled the entire spinal cord segment, thus, forming new neuronal relay circuits and improving neural electrophysiological function and motor function (Lu et al., 2012; Rosenzweig et al., 2018; Kumamaru et al., 2019; Ceto et al., 2020). Although changes in the fibrotic scar have not been directly evaluated after NSC grafting, the regenerated axons passing through the injured core and filling the entire spinal cord segment suggest that NSCs may be able to overcome the inhibitory effect of the fibrotic scar (Lu et al., 2012; Rosenzweig et al., 2018). Nevertheless, Paul Lu et al. reported that human induced pluripotent stem cell (iPSC)derived NSC grafts transplanted into the injured site differentiate into neurons that are distributed through most of the injured site, while a rift forms at the middle of the graft and is filled with collagen, thus, impairing axonal regeneration ( $\mathrm{Lu}$ et al., 2014). This process results in the disconnection of neuronal relay circuits formed by the graft, and improved functional recovery is not achieved ( $\mathrm{Lu}$ et al., 2014). These results indicate the need for additional studies of the changes in the fibrotic scar after NSC graft and its mechanism to provide the basis for the treatment of SCI by combining NSC grafts with inhibition of fibrotic scarring. Furthermore, Ephron S. Rosenzweig et al. reported that an injection of chondroitinase below the lesion to degrade CSPGs promotes axonal regeneration and improves motor function after SCI in primates, while the changes in the fibrotic scar are unknown (Rosenzweig et al., 2019). Studies targeting extracellular matrix molecules to treat SCI may focus on the effect on fibrotic scar, which is expected to provide a basis for the treatment of SCI.

Overall, fibrotic scar formation after SCI is a complex process that includes the migration, proliferation, and ECM deposition of fibroblasts (Figure 3). The application of drugs and

\section{REFERENCES}

Anderson, M., Burda, J., Ren, Y., Ao, Y., O’Shea, T., Kawaguchi, R., et al. (2016). Astrocyte scar formation aids central nervous system axon regeneration. Nature 532, 195-200. doi: 10.1038/nature17623

Armulik, A., Genové, G., Mäe, M., Nisancioglu, M., Wallgard, E., Niaudet, C., et al. (2010). Pericytes regulate the blood-brain barrier. Nature 468, 557-561. doi: 10.1038 /nature09522

Bataller, R., and Brenner, D. (2005). Liver fibrosis. J. Clin. Invest. 115, 209-218. doi: $10.1172 / \mathrm{JCI} 24282$

Bellver-Landete, V., Bretheau, F., Mailhot, B., Vallières, N., Lessard, M., Janelle, M., et al. (2019). Microglia are an essential component of the neuroprotective scar that forms after spinal cord injury. Nat. Commun. 10:518. doi: 10.1038/s41467-019-08446-0

Bhattacharyya, S., Kelley, K., Melichian, D., Tamaki, Z., Fang, F., Su, Y., et al. (2013). Toll-like receptor 4 signaling augments transforming growth factor$\beta$ responses: a novel mechanism for maintaining and amplifying fibrosis in scleroderma. Am. J. Pathol. 182, 192-205. doi: 10.1016/j.ajpath.2012. 09.007

Bondjers, C., He, L., Takemoto, M., Norlin, J., Asker, N., Hellström, M., et al. (2006). Microarray analysis of blood microvessels from PDGF-B and PDGFRbeta mutant mice identifies novel markers for brain pericytes. FASEB J. 20, 1703-1705. doi: 10.1096/fj.05-4944fje transgenic treatment strategies in an animal model of SCI has provided positive results that appropriately attenuating fibrotic scars contribute to axonal regeneration and the recovery of sensorimotor function (Table 1; Klapka et al., 2005; Zhu et al., 2015a; Yokota et al., 2017; Dias et al., 2018; Wang et al., 2018a,b). In addition, recent research advances in the identification and spatiotemporal distribution of fibrotic scar-forming fibroblasts after SCI have provided more evidence for the fibrotic scar as a treatment target after SCI. Further explorations of the cellular and molecular mechanisms underlying the formation of fibrotic scar after SCI are very important, which is expected to accelerate translation from the laboratory to the clinic.

\section{AUTHOR CONTRIBUTIONS}

JJ, MZ, and LC contributed to the design and revision of the review. ZL and SY edited and revised the manuscript. DT, XY, $\mathrm{XH}$, and YL prepared the figures and table. All authors read and approved the final manuscript.

\section{FUNDING}

This study was supported by the Key Research and Development Projects of Anhui Province (Grant number 202004j07020042), the National Natural Science Foundation of China (Grant numbers 81801220 and 81671204), and the Provincial Natural Science Research Key Project of Colleges and Universities of Anhui Province (Grant number KJ2019A0257).

\section{ACKNOWLEDGMENTS}

We acknowledge the experimental platform provided by the Scientific Research and Experiment Center of the Second Hospital of Anhui Medical University.
Buechler, M., Fu, W., and Turley, S. (2021). Fibroblast-macrophage reciprocal interactions in health, fibrosis, and cancer. Immunity 54, 903-915. doi: 10.1016/j.immuni.2021.04.021

Bundesen, L., Scheel, T., Bregman, B., and Kromer, L. (2003). Ephrin-B2 and EphB2 regulation of astrocyte-meningeal fibroblast interactions in response to spinal cord lesions in adult rats. J. Neurosci. 23, 7789-7800. doi: 10.1523/JNEUROSCI.23-21-07789.2003

Buss, A., Pech, K., Kakulas, B., Martin, D., Schoenen, J., Noth, J., et al. (2007). Growth-modulating molecules are associated with invading Schwann cells and not astrocytes in human traumatic spinal cord injury. Brain 130, 940-953. doi: 10.1093/brain/awl374

Cai, B., Dongiovanni, P., Corey, K., Wang, X., Shmarakov, I., Zheng, Z., et al. (2020). Macrophage MerTK promotes liver fibrosis in nonalcoholic steatohepatitis. Cell Metab. 31, 406-421.e407. doi: 10.1016/j.cmet.2019. 11.013

Camand, E., Morel, M., Faissner, A., Sotelo, C., and Dusart, I. (2004). Long-term changes in the molecular composition of the glial scar and progressive increase of serotoninergic fibre sprouting after hemisection of the mouse spinal cord. Eur. J. Neurosci. 20, 1161-1176. doi: 10.1111/j.1460-9568.2004.03558.x

Ceto, S., Sekiguchi, K., Takashima, Y., Nimmerjahn, A., and Tuszynski, M. (2020). Neural stem cell grafts form extensive synaptic networks that integrate with host circuits after spinal cord injury. Cell Stem Cell 27, 430-440.e435. doi: $10.1016 /$ j.stem.2020.07.007 
Chan, C., Harley, I., Pfluger, P., Trompette, A., Stankiewicz, T., Allen, J., et al. (2021). A BAFF/APRIL axis regulates obesogenic diet-driven weight gain. Nat. Commun. 12:2911. doi: 10.1038/s41467-021-23084-1

Cooper, J., Jeong, S., McGuire, T., Sharma, S., Wang, W., Bhattacharyya, S., et al. (2018). Fibronectin EDA forms the chronic fibrotic scar after contusive spinal cord injury. Neurobiol. Dis. 116, 60-68. doi: 10.1016/j.nbd.2018. 04.014

D'Ambrosi, N., and Apolloni, S. (2020). Fibrotic scar in neurodegenerative diseases. Front. Immunol. 11:1394. doi: 10.3389/fimmu.2020. 01394

Daneman, R., Zhou, L., Kebede, A., and Barres, B. (2010). Pericytes are required for blood-brain barrier integrity during embryogenesis. Nature 468, 562-566. doi: 10.1038 /nature 09513

Darby, I., and Hewitson, T. (2007). Fibroblast differentiation in wound healing and fibrosis. Int. Rev. Cytol. 257, 143-179. doi: 10.1016/S0074-7696(07) 57004-X

DasGupta, R., and Fuchs, E. (1999). Multiple roles for activated LEF/TCF transcription complexes during hair follicle development and differentiation. Development 126, 4557-4568. doi: 10.1242/dev.126. 20.4557

Dias, D., and Göritz, C. (2018). Fibrotic scarring following lesions to the central nervous system. Matrix. Biol. 561-570. doi: 10.1016/j.matbio.2018. 02.009

Dias, D., Kim, H., Holl, D., Werne Solnestam, B., Lundeberg, J., Carlén, M., et al. (2018). Reducing pericyte-derived scarring promotes recovery after spinal cord injury. Cell 173, 153-165.e122. doi: 10.1016/j.cell.2018. 02.004

Dorrier, C., Aran, D., Haenelt, E., Sheehy, R., Hoi, K., Pintarić, L., et al. (2021). CNS fibroblasts form a fibrotic scar in response to immune cell infiltration. Nat. Neurosci. 24, 234-244. doi: 10.1038/s41593-02000770-9

Fehlberg, C., and Lee, J. (2021). Fibrosis in the central nervous system: from the meninges to the vasculature. Cell Tissue Res. doi: 10.1007/s00441-02103491-y. [Epub ahead of print].

Funk, L., Hackett, A., Bunge, M., and Lee, J. (2016). Tumor necrosis factor superfamily member APRIL contributes to fibrotic scar formation after spinal cord injury. J. Neuroinflammation 13:87. doi: 10.1186/s12974-016$0552-4$

Goldshmit, Y., Galea, M., Wise, G., Bartlett, P., and Turnley, A. (2004). Axonal regeneration and lack of astrocytic gliosis in EphA4-deficient mice. J. Neurosci. 24, 10064-10073. doi: 10.1523/JNEUROSCI.2981-04.2004

Göritz, C., Dias, D., Tomilin, N., Barbacid, M., Shupliakov, O., and Frisén, J. (2011). A pericyte origin of spinal cord scar tissue. Science 333, 238-242. doi: 10.1126/science. 1203165

Hakuno, D., Kimura, N., Yoshioka, M., Mukai, M., Kimura, T., Okada, Y., et al. (2010). Periostin advances atherosclerotic and rheumatic cardiac valve degeneration by inducing angiogenesis and MMP production in humans and rodents. J. Clin. Invest. 120, 2292-2306. doi: 10.1172/JCI40973

Hara, M., Kobayakawa, K., Ohkawa, Y., Kumamaru, H., Yokota, K., Saito, T., et al. (2017). Interaction of reactive astrocytes with type I collagen induces astrocytic scar formation through the integrin- $\mathrm{N}$-cadherin pathway after spinal cord injury. Nat. Med. 23, 818-828. doi: 10.1038/nm.4354

Hellal, F., Hurtado, A., Ruschel, J., Flynn, K., Laskowski, C., Umlauf, M., et al. (2011). Microtubule stabilization reduces scarring and causes axon regeneration after spinal cord injury. Science 331, 928-931. doi: $10.1126 /$ science. 1201148

Hermanns, S., Klapka, N., and Müller, H. (2001). The collagenous lesion scaran obstacle for axonal regeneration in brain and spinal cord injury. Restor. Neurol. Neurosci. 19, 139-148. Available online at: https://content.iospress. com/articles/restorative-neurology-and-neuroscience/rnn00172

Herrmann, J., Imura, T., Song, B., Qi, J., Ao, Y., Nguyen, T., et al. (2008). STAT3 is a critical regulator of astrogliosis and scar formation after spinal cord injury. J. Neurosci. 28, 7231-7243. doi: 10.1523/JNEUROSCI.1709-08.2008

Herrmann, J., Shah, R., Chan, A., and Zheng, B. (2010). EphA4 deficient mice maintain astroglial-fibrotic scar formation after spinal cord injury. Exp. Neurol. 223, 582-598. doi: 10.1016/j.expneurol.2010.02.005

Humphreys, B., Lin, S., Kobayashi, A., Hudson, T., Nowlin, B., Bonventre, J., et al. (2010). Fate tracing reveals the pericyte and not epithelial origin of myofibroblasts in kidney fibrosis. Am. J. Pathol. 176, 85-97. doi: 10.2353/ajpath.2010.090517

Izuhara, K., Conway, S., Moore, B., Matsumoto, H., Holweg, C., Matthews, J., et al. (2016). Roles of periostin in respiratory disorders. Am. J. Respir. Crit. Care Med. 193, 949-956. doi: 10.1164/rccm.201510-2032PP

Kalluri, R., and Zeisberg, M. (2006). Fibroblasts in cancer. Nature Rev. Canc. 6, 392-401. doi: 10.1038/nrc1877

Kawano, H., Kimura-Kuroda, J., Komuta, Y., Yoshioka, N., Li, H., Kawamura, K., et al. (2012). Role of the lesion scar in the response to damage and repair of the central nervous system. Cell Tissue Res. 349, 169-180. doi: 10.1007/s00441-012-1336-5

Klapka, N., Hermanns, S., Straten, G., Masanneck, C., Duis, S., Hamers, F., et al. (2005). Suppression of fibrous scarring in spinal cord injury of rat promotes long-distance regeneration of corticospinal tract axons, rescue of primary motoneurons in somatosensory cortex and significant functional recovery. Eur. J. Neurosci. 22, 3047-3058. doi: 10.1111/j.1460-9568.2005. 04495.x

Kobayakawa, K., Ohkawa, Y., Yoshizaki, S., Tamaru, T., Saito, T., Kijima, K., et al. (2019). Macrophage centripetal migration drives spontaneous healing process after spinal cord injury. Sci. Adv. 5:eaav5086. doi: 10.1126/sciadv. aav5086

Kostyk, S., Popovich, P., Stokes, B., Wei, P., and Jakeman, L. (2008). Robust axonal growth and a blunted macrophage response are associated with impaired functional recovery after spinal cord injury in the MRL/MpJ mouse. Neuroscience 156, 498-514. doi: 10.1016/j.neuroscience.2008. 08.013

Krenning, G., Zeisberg, E., and Kalluri, R. (2010). The origin of fibroblasts and mechanism of cardiac fibrosis. J. Cell. Physiol. 225, 631-637. doi: $10.1002 /$ jcp. 22322

Kumamaru, H., Lu, P., Rosenzweig, E., Kadoya, K., and Tuszynski, M. (2019). Regenerating corticospinal axons innervate phenotypically appropriate neurons within neural stem cell grafts. Cell Rep. 26, 2329-2339.e2324. doi: 10.1016/j.celrep.2019.01.099

Lang, B., Cregg, J., DePaul, M., Tran, A., Xu, K., Dyck, S., et al. (2015). Modulation of the proteoglycan receptor PTP $\sigma$ promotes recovery after spinal cord injury. Nature 518, 404-408. doi: 10.1038/nature 13974

Lederer, D., and Martinez, F. (2018). Idiopathic pulmonary fibrosis. N. Engl. J. Med. 378, 1811-1823. doi: 10.1056/NEJMra 1705751

Lee, S., and Kalluri, R. (2010). Mechanistic connection between inflammation and fibrosis. Kidney Int. Suppl. 78:S22-S26. doi: 10.1038/ki.2010.418

Li, H., Kong, W., Chambers, C., Yu, D., Ganea, D., Tuma, R., et al. (2018). The non-psychoactive phytocannabinoid cannabidiol (CBD) attenuates pro-inflammatory mediators, $\mathrm{T}$ cell infiltration, and thermal sensitivity following spinal cord injury in mice. Cell. Immunol. 329, 1-9. doi: 10.1016/j.cellimm.2018.02.016

Li, Y., He, X., Kawaguchi, R., Zhang, Y., Wang, Q., Monavarfeshani, A., et al. (2020). Microglia-organized scar-free spinal cord repair in neonatal mice. Nature 587, 613-618. doi: 10.1038/s41586-0202795-6

Li, Z., Zheng, M., Yu, S., Yao, F., Luo, Y., Liu, Y., et al. (2021). M2 macrophages promote PDGFR $\beta+$ pericytes migration after spinal cord injury in mice via PDGFB/PDGFR $\beta$ pathway. Front. Pharmacol. 12:670813. doi: 10.3389/fphar.2021. 670813

Lie, D., Colamarino, S., Song, H., Désiré, L., Mira, H., Consiglio, A., et al. (2005). Wnt signalling regulates adult hippocampal neurogenesis. Nature 437, 1370-1375. doi: 10.1038/nature04108

Lin, S., Kisseleva, T., Brenner, D., and Duffield, J. (2008). Pericytes and perivascular fibroblasts are the primary source of collagen-producing cells in obstructive fibrosis of the kidney. Am. J. Pathol. 173, 1617-1627. doi: 10.2353/ajpath.2008.080433

Liu, Z., Zhang, H., Xia, H., Wang, B., Zhang, R., Zeng, Q., et al. (2019). CD8 T cellderived perforin aggravates secondary spinal cord injury through destroying the blood-spinal cord barrier. Biochem. Biophys. Res. Commun. 512, 367-372. doi: 10.1016/j.bbrc.2019.03.002

Lu, P., Wang, Y., Graham, L., McHale, K., Gao, M., Wu, D., et al. (2012). Longdistance growth and connectivity of neural stem cells after severe spinal cord injury. Cell 150, 1264-1273. doi: 10.1016/j.cell.2012.08.020 
Lu, P., Woodruff, G., Wang, Y., Graham, L., Hunt, M., Wu, D., et al. (2014). Longdistance axonal growth from human induced pluripotent stem cells after spinal cord injury. Neuron 83, 789-796. doi: 10.1016/j.neuron.2014.07.014

Mack, M. (2018). Inflammation and fibrosis. Matrix. Biol. 106-121. doi: 10.1016/j.matbio.2017.11.010

Metz, G., Curt, A., van de Meent, H., Klusman, I., Schwab, M., and Dietz, V. (2000). Validation of the weight-drop contusion model in rats: a comparative study of human spinal cord injury. J. Neurotrauma 17, 1-17. doi: 10.1089/neu.2000.17.1

Norenberg, M., Smith, J., and Marcillo, A. (2004). The pathology of human spinal cord injury: defining the problems. J. Neurotrauma 21, 429-440. doi: $10.1089 / 089771504323004575$

Okada, M., Miyamoto, O., Shibuya, S., Zhang, X., Yamamoto, T., and Itano, T. (2007). Expression and role of type I collagen in a rat spinal cord contusion injury model. Neurosci. Res. 58, 371-377. doi: 10.1016/j.neures.2007.04.009

Pardali, E., Sanchez-Duffhues, G., Gomez-Puerto, M., and Ten Dijke, P. (2017). TGF- $\beta$-induced endothelial-mesenchymal transition in fibrotic diseases. Int. J. Mol. Sci. 18:2157. doi: 10.3390/ijms18102157

Ren, Z., Chen, X., Yang, J., Kress, B., Tong, J., Liu, H., et al. (2013). Improved axonal regeneration after spinal cord injury in mice with conditional deletion of ephrin B2 under the GFAP promoter. Neuroscience 241, 89-99. doi: 10.1016/j.neuroscience.2013.03.010

Renault-Mihara, F., Mukaino, M., Shinozaki, M., Kumamaru, H., Kawase, S., Baudoux, M., et al. (2017). Regulation of RhoA by STAT3 coordinates glial scar formation. J. Cell. Biol. 216, 2533-2550. doi: 10.1083/jcb.201610102

Rockey, D., Bell, P., and Hill, J. (2015). Fibrosis-a common pathway to organ injury and failure. N. Engl. J. Med. 372, 1138-1149. doi: 10.1056/NEJMra1300575

Rosenzweig, E., Brock, J., Lu, P., Kumamaru, H., Salegio, E., Kadoya, K., et al. (2018). Restorative effects of human neural stem cell grafts on the primate spinal cord. Nat. Med. 24, 484-490. doi: 10.1038/nm.4502

Rosenzweig, E., Salegio, E., Liang, J., Weber, J., Weinholtz, C., Brock, J., et al. (2019). Chondroitinase improves anatomical and functional outcomes after primate spinal cord injury. Nat. Neurosci. 22, 1269-1275. doi: 10.1038/s41593-019-0424-1

Rudge, J., and Silver, J. (1990). Inhibition of neurite outgrowth on astroglial scars in vitro. J. Neurosci. 10, 3594-3603. doi: 10.1523/JNEUROSCI.10-11-03594.1990

Ruschel, J., Hellal, F., Flynn, K., Dupraz, S., Elliott, D., Tedeschi, A., et al. (2015). Axonal regeneration. Systemic administration of epothilone B promotes axon regeneration after spinal cord injury. Science 348, 347-352. doi: 10.1126/science.aaa2958

Schiwy, N., Brazda, N., and Müller, H. (2009). Enhanced regenerative axon growth of multiple fibre populations in traumatic spinal cord injury following scar-suppressing treatment. Eur. J. Neurosci. 30, 1544-1553. doi: $10.1111 /$ j.1460-9568.2009.06929.x

Shearer, M., and Fawcett, J. (2001). The astrocyte/meningeal cell interfacea barrier to successful nerve regeneration? Cell Tissue Res. 305, 267-273. doi: 10.1007/s004410100384

Slezak, M., Göritz, C., Niemiec, A., Frisén, J., Chambon, P., Metzger, D., et al. (2007). Transgenic mice for conditional gene manipulation in astroglial cells. Glia 55, 1565-1576. doi: 10.1002/glia.20570

Soderblom, C., Luo, X., Blumenthal, E., Bray, E., Lyapichev, K., Ramos, J., et al. (2013). Perivascular fibroblasts form the fibrotic scar after contusive spinal cord injury. J. Neurosci. 33, 13882-13887. doi: 10.1523/JNEUROSCI.252413.2013

Sofroniew, M. (2018). Dissecting spinal cord regeneration. Nature 557, 343-350. doi: 10.1038/s41586-018-0068-4

Srinivas, S., Watanabe, T., Lin, C., William, C., Tanabe, Y., Jessell, T., et al. (2001). Cre reporter strains produced by targeted insertion of EYFP and ECFP into the ROSA26 locus. BMC Dev. Biol. 1:4. doi: 10.1186/1471213X-1-4

Sugiyama, A., Kanno, K., Nishimichi, N., Ohta, S., Ono, J., Conway, S., et al. (2016). Periostin promotes hepatic fibrosis in mice by modulating hepatic stellate cell activation via $\alpha$ integrin interaction. J. Gastroenterol. 51, 1161-1174. doi: 10.1007/s00535-016-1206-0

Sundberg, C., Ivarsson, M., Gerdin, B., and Rubin, K. (1996). Pericytes as collagenproducing cells in excessive dermal scarring. Lab. Invest. 74, 452-466.

Tran, A., Warren, P., and Silver, J. (2018). The Biology of Regeneration Failure and Success After Spinal Cord Injury. Physiol. Rev. 98, 881-917. doi: 10.1152/physrev.00017.2017
Travers, J., Kamal, F., Robbins, J., Yutzey, K., and Blaxall, B. (2016). Cardiac fibrosis: the fibroblast awakens. Circ. Res. 118, 1021-1040. doi: 10.1161/CIRCRESAHA.115.306565

Vanlandewijck, M., He, L., Mäe, M., Andrae, J., Ando, K., Del Gaudio, F., et al. (2018). A molecular atlas of cell types and zonation in the brain vasculature. Nature 554, 475-480. doi: 10.1038/nature25739

Wang, H., Wang, K., Zhong, X., Qiu, W., Dai, Y., Wu, A., et al. (2012). Cerebrospinal fluid BAFF and APRIL levels in neuromyelitis optica and multiple sclerosis patients during relapse. J. Clin. Immunol. 32, 1007-1011. doi: 10.1007/s10875-012-9709-9

Wang, W., Liu, R., Su, Y., Li, H., Xie, W., and Ning, B. (2018a). MicroRNA-21$5 p$ mediates TGF- $\beta$-regulated fibrogenic activation of spinal fibroblasts and the formation of fibrotic scars after spinal cord injury. Int. J. Biol. Sci. 14, 178-188. doi: $10.7150 /$ ijbs. 24074

Wang, W., Tang, S., Li, H., Liu, R., Su, Y., Shen, L., et al. (2018b). MicroRNA-21a5 p promotes fibrosis in spinal fibroblasts after mechanical trauma. Exp. Cell Res. 370, 24-30. doi: 10.1016/j.yexcr.2018.06.002

Wang, X., Cao, K., Sun, X., Chen, Y., Duan, Z., Sun, L., et al. (2015). Macrophages in spinal cord injury: phenotypic and functional change from exposure to myelin debris. Glia 63, 635-651. doi: 10.1002/glia. 22774

Wang, Y., Plane, J., Jiang, P., Zhou, C., and Deng, W. (2011). Concise review: quiescent and active states of endogenous adult neural stem cells: identification and characterization. Stem Cells 29, 907-912. doi: 10.1002/stem.644

Wanner, I., Anderson, M., Song, B., Levine, J., Fernandez, A., Gray-Thompson, Z., et al. (2013). Glial scar borders are formed by newly proliferated, elongated astrocytes that interact to corral inflammatory and fibrotic cells via STAT3dependent mechanisms after spinal cord injury. J. Neurosci. 33, 12870-12886. doi: 10.1523/JNEUROSCI.2121-13.2013

Weldon, A., Moldovan, I., Cabling, M., Hernandez, E., Hsu, S., Gonzalez, J., et al. (2015). Surface APRIL is elevated on myeloid cells and is associated with disease activity in patients with rheumatoid arthritis. J. Rheumatol. 42, 749-759. doi: 10.3899/jrheum.140630

Wu, J., Lu, B., Yang, R., Chen, Y., Chen, X., and Li, Y. (2021). EphB2 knockdown decreases the formation of astroglial-fibrotic scars to promote nerve regeneration after spinal cord injury in rats. CNS Neurosci. Ther. 27, 714-724. doi: $10.1111 / \mathrm{cns} .13641$

Xie, C., Shen, X., Xu, X., Liu, H., Li, F., Lu, S., et al. (2020). Astrocytic YAP promotes the formation of glia scars and neural regeneration after spinal cord injury. J. Neurosci. 40, 2644-2662. doi: 10.1523/JNEUROSCI.222919.2020

Yamagami, T., Pleasure, D., Lam, K., and Zhou, C. (2018). Transient activation of Wnt/ $\beta$-catenin signaling reporter in fibrotic scar formation after compression spinal cord injury in adult mice. Biochem. Biophys. Res. Commun. 496, 1302-1307. doi: 10.1016/j.bbrc.2018.02.004

Yamaguchi, Y. (2014). Periostin in skin tissue skin-related diseases. Allergol. Int. 63, 161-170. doi: 10.2332/allergolint.13-RAI-0685

Yata, Y., Scanga, A., Gillan, A., Yang, L., Reif, S., Breindl, M., et al. (2003). DNase I-hypersensitive sites enhance alpha1(I) collagen gene expression in hepatic stellate cells. Hepatology 37, 267-276. doi: 10.1053/jhep.2003. 50067

Yokota, K., Kobayakawa, K., Saito, T., Hara, M., Kijima, K., Ohkawa, Y., et al. (2017). Periostin promotes scar formation through the interaction between pericytes and infiltrating monocytes/macrophages after spinal cord injury. Am. J. Pathol. 187, 639-653. doi: 10.1016/j.ajpath.2016. 11.010

Zeisberg, M., and Kalluri, R. (2004). The role of epithelial-to-mesenchymal transition in renal fibrosis. J. Mol. Med. (Berl.) 82, 175-181. doi: 10.1007/s00109-003-0517-9

Zhang, S., Geddes, J., Owens, J., and Holmberg, E. (2005). X-irradiation reduces lesion scarring at the contusion site of adult rat spinal cord. Histol. Histopathol. 20, 519-530. doi: 10.14670/HH-20.519

Zhou, X., Wahane, S., Friedl, M., Kluge, M., Friedel, C., Avrampou, K., et al. (2020). Microglia and macrophages promote corralling, wound compaction and recovery after spinal cord injury via Plexin-B2. Nat. Neurosci. 23, 337-350. doi: 10.1038/s41593-020-0597-7

Zhu, Y., Soderblom, C., Krishnan, V., Ashbaugh, J., Bethea, J., and Lee, J. (2015a). Hematogenous macrophage depletion reduces the fibrotic scar and 
increases axonal growth after spinal cord injury. Neurobiol. Dis. 74, 114-125. doi: 10.1016/j.nbd.2014.10.024

Zhu, Y., Soderblom, C., Trojanowsky, M., Lee, D., and Lee, J. (2015b). Fibronectin matrix assembly after spinal cord injury. J. Neurotrauma 32, 1158-1167. doi: 10.1089/neu.2014.3703

Zivkovic, S., Ayazi, M., Hammel, G., and Ren, Y. (2021). For better or for worse: a look into neutrophils in traumatic spinal cord injury. Front. Cell. Neurosci. 15:648076. doi: 10.3389/fncel.2021. 648076

Conflict of Interest: The authors declare that the research was conducted in the absence of any commercial or financial relationships that could be construed as a potential conflict of interest.
Publisher's Note: All claims expressed in this article are solely those of the authors and do not necessarily represent those of their affiliated organizations, or those of the publisher, the editors and the reviewers. Any product that may be evaluated in this article, or claim that may be made by its manufacturer, is not guaranteed or endorsed by the publisher.

Copyright $\odot 2021 \mathrm{Li}, \mathrm{Yu}, \mathrm{Hu}, \mathrm{Li}$, You, Tian, Cheng, Zheng and Jing. This is an open-access article distributed under the terms of the Creative Commons Attribution License (CC BY). The use, distribution or reproduction in other forums is permitted, provided the original author(s) and the copyright owner(s) are credited and that the original publication in this journal is cited, in accordance with accepted academic practice. No use, distribution or reproduction is permitted which does not comply with these terms. 